\title{
Brain stem and cerebellar dysfunction with Legionnaires' disease
}

\author{
PHILIP CH BAKER,* TREVOR RP PRICE, $\dagger$ COLIN D ALLEN* \\ From the Section of Neurology* and the Department of Psychiatry, $\dagger$ Dartmouth-Hitchcock Medical Center, \\ Hanover, New Hampshire, USA
}

SUMMARY A 37-year-old man under treatment for manic-depressive illness developed pneumonia identified as Legionnaires' disease accompanied by a severe neurological disorder with profound dysarthria, ataxia, gaze paralysis, and downbeat nystagmus. At review six months later, he has made only a partial recovery with persisting limb and gait ataxia. Difficulties in diagnosing neurological complications of Legionnaires' disease in a patient with a psychiatric disorder requiring psychotropic medication are discussed.

Legionnaires' disease is a potentially fatal, infectious disease caused by the gram-negative bacillus Legionella pneumophila. A reversible diffuse encephalopathy is the most common neurological complication but focal involvement with brain stem and cerebellar dysfunction may also occur with slow and incomplete recovery. This report describes a patient with Legionnaires' pneumonia complicated by gaze paralysis and unusually severe and persistent cerebellar-rubral dysfunction.

\section{Case report}

A 37-year-old man with a ten year history of bipolar illness was admitted with a three week history of increasingly severe manic behaviour. Over the previous year he had been clinically stable on lithium carbonate $2400 \mathrm{mg} /$ day. He was treated with increasing doses of haloperidol up to $70 \mathrm{mg} / \mathrm{day}$, and after one week he developed moderate limb rigidity, hand tremor and drooling. Benztropine was prescribed, and haloperidol was reduced in dosage and then stopped. In order to control agitation and combative behaviour he was given diphenhydramine and secobarbitol; this was followed by seven ECT treatments given over three days. He became less manic, and although extrapyramidal signs diminished, he still showed mild rigidity of his limbs, and a resting tremor of his hands.

Six days after his last ECT, he became confused and

Address for reprint requests: Dr Philip CH Baker, Section of Neurology, Dartmouth-Hitchcock Medical Center, Hanover, New Hampshire 03755, USA.

Received 8 August 1981, accepted 1 September 1981 delirious and the following day his temperature rose to $40^{\circ} \mathrm{C}$. Physical examination and chest radiograph revealed consolidation of the right lower lobe and intramuscular penicillin was started. Two days later he was still febrile and agitated and his speech was barely intelligible due to a severe dysarthria. He had striking downbeat nystagmus and was unable to voluntarily elevate or laterally deviate his eyes; however full horizontal movement could be induced by cold water caloric testing. The remainder of his cranial nerves were normal and his optic fundi were normal. While resting undisturbed there was no tremor or abnormal movement, but on arousal and on attempting to perform activity to command, a severe, irregular, high-amplitude flailing movement of his arms would develop frequently causing him to strike his face and head with his hands. Both legs were also very ataxic and he was unable to stand. Muscle strength, however, was normal, limb reflexes were physiological and plantar responses flexor.

Routine blood tests were normal apart from a WBC of $20.6 \times 10^{9} / 1(92 \%$ neutrophils, $8 \%$ bands $)$; plasma lithium was $1 \cdot 2 \mathrm{mmol} / \mathrm{l}$ (therapeutic range $0 \cdot 7-1 \cdot 4$ ); spinal fluid was under a pressure of $216 \mathrm{~mm}$ of $\mathrm{CSF}$, and contained $1 \mathrm{RBC} / \mathrm{mm}^{3}, 3 \mathrm{WBC} / \mathrm{mm}^{3}$, protein $0.36 \mathrm{~g} / \mathrm{l}$ and glucose $4.3 \mathrm{mmol} / \mathrm{l}$. Cultures of spinal fluid, blood, urine and sputum were all negative and microscopy of a transtracheal aspirate showed many polymorphs but no organisms. Computed tomography and brain stem auditory-evoked responses were normal. Antibody titres against Legionnaires' disease antigen were $1: 128$ on the third day of his pneumonic illness and had increased fourfold to $1: 512$ four weeks later, a rise consistent with recent Legionnaires' disease.

He was treated with erythromycin, $500 \mathrm{mg}$ every six hours, and his temperature dropped to normal over two 1054 
days. Blood levels of barbiturate and lithium were within the therapeutic range throughout the illness and these drugs were withdrawn over several days. The severely disorganised movements of his hands and arms became less violent and with improved cooperation it became apparent that these represented a grossly exaggerated cerebellar or rubral ataxia. Bouts of hiccoughing occurred in the early stages of his illness, but had subsided after a week; as his pneumonia cleared, he became more responsive and at the end of one week he was cognitively intact. He continued to show downbeat nystagmus for a further four weeks with gradual return of full conjugate eye movement; however, dysarthria persisted and there was only minor improvement of his limb ataxia such that at the end of the first month he was able to take a few unsteady steps with a walker.

Improvement since then has been minimal. Recurrence of his bipolar illness has required the reintroduction of lithium and carbamazepine with only partial improvement, and he has received further ECT to control periods of mania. Atazia and dysathria have become noticeably worse at the times of instability of his psychiatric disorder with improvement of these signs after ECT. At one year follow-up he is still severely disabled by limb and gait ataxia and he cannot stand or walk without the support of a walking frame; his speech remains slurred but intelligible and he requires assistance with feeding and dressing.

\section{Discussion}

Headache, delirium and drowsiness are commonly reported features of Legionnaires' disease. Disturbance of gait and hallucinations are also often described, ${ }^{1-6}$ and occasional patients have been reported with upper limb ataxia and tremor $;^{3-5}$ 7 increased muscle tone, hyperactive tendon reflexes and extensor plantar responses; memory deficit and personality change; seizures; ${ }^{34}$ neck stiffness; cranial nerve palsies ${ }^{2}$ and, in a single case, transverse myelopathy. ${ }^{4}$ Neurological symptoms usually occur early in the course of the illness and delirium may develop before the fever and signs of pneumonia appear.

The neurological disorder seen in this patient included delirium, downbeat nystagmus, supranuclear horizontal and vertical gaze paralysis, and a coarse high-amplitude action tremor with limb and gait ataxia. This complex of signs suggested diffuse cerebral disturbance with major involvement of brainstem and cerebellar pathways. The presence of supranuclear gaze paralysis indicated bilateral dysfunction above the level of the third nerve nuclei. Lesions of the dentatothalamic and rubrothalamic tracts may characteristically produce ataxia and intention tremor of the type that this patient showed and, therefore, it is possible to account for many of his neurological signs by bilateral involvement of the upper brainstem at or rostral to the junction of midbrain and diencepahlon. Downbeat nystagmus however is usually a feature of disease at a lower level (pons) indicating that there was more widespread dysfunction in the brainstem.

The pathogenesis of the neurological complications of Legionnaires' disease remains unknown. Spinal fluid examination is usually normal, as it was in this case, suggesting that a direct bacterial meningoencephalitis does not occur. Other possibilities include a vasculitis or a toxic effect, and it has been postulated that a lipopolysaccharide endotoxin ${ }^{8}$ may enter the CNS; measurement of antibody titres in spinal fluid might confirm this hypothesis.

Diagnosis in this case was complicated by the patient's previous drug therapy. The extrapyramidal side effects of haloperidol were resolving with treatment at the time limb gait ataxia developed. Initially these new features were also attributed to drug toxicity, since it has been reported that a combination of lithium and haloperidol, or lithium alone, may cause cerebellar dysfunction with permanent brain damage in occasional patients, ${ }^{9}$ although other studies have questioned this conclusion. ${ }^{1011}$ However, there were no certain clinical features of lithium toxicity and serum levels of this drug and of barbiturates were never within the toxic range. Ataxia, dysarthria, and downbeat nystagmus first became evident two to three days after the development of pneumonia and it seems clear that these neurological features were associated with his acute systemic illness, confirmed later by serological tests to be Legionnaires' disease. Whether psychiatric illness, psychotropic medications or previous ECT treatment increases the susceptibility of subjects to develop CNS complications from Legionella infection is at present unknown. There is no available evidence to suggest that medications predispose patients to this effect but ECT has been reported to increase blood-brain barrier permeability ${ }^{12}$ which might lead to increased concentrations of the putative Legionnaires' toxin in brain tissue.

In non-fatal cases of Legionnaires' disease, signs of diffuse cerebral dysfunction usually resolve rapidly and completely, although exceptions have been reported; Gregory et $a^{5}$ described one patient who showed serious residual memory impairment. Cerebellar dysfunction usually improves but may take months to resolve; Friedmen, ${ }^{4}$ Gregory et al, ${ }^{5}$ and Shetty et $\mathrm{al}^{7}$ have reported patients with persisting disorders of speech and gait. The patient described in this report is unusual in having shown only marginal improvement in his baseline neurological function six months after recovery from pneumonia. It appears likely that he will have major permanent cerebellar dysfunction. 
This case illustrates the potential severity and persistence of neurologic complications of Legionnaires' disease. It also demonstrates the difficulty of diagnosing and managing these complications in a patient with a relapsing psychiatric illness and emphasises the danger of attributing unusual signs and symptoms to psychotropic drugs alone.

\section{References}

${ }^{1}$ Terranova W, Cohen ML, Frazer DW. 1974 outbreak of Legionnaires' disease diagnosed in 1977-clinical and epidemiologic features. Lancet 1978;15:122-4.

${ }^{2}$ Lattimer GL, Rhodes LV III. Legionnaires' disease. Clinical findings and one year follow-up. JAMA 1978;240:1169-71.

${ }^{3}$ Lees AW, Tyrell WF. Severe cerebral disturbance in Legionnaires' disease. Lancet 1978;3:1336-7.

${ }^{4}$ Friedman MH. Legionnaires' disease in non-Legionnaires'; a report of five cases. Ann Int Med 1978; 88:294-302.

${ }^{5}$ Gregory DW, Schaffner W, Alford RH, Kaiser AB, McGee ZA. Sporadic cases of Legionnaires' disease: the expanding clinical spectrum. Ann Int Med 1979; 90:518-21.

${ }^{6}$ Keys TF. Legionnaires' disease; a review of the epidemiology and clinical manifestations of a newly recognised infection. Mayo Clinic Proc 1980;55: 129-37.

${ }^{7}$ Shetty KR, Cilyo CL, Starr BD, Harter DH. Legionnaires' disease with profound cerebellar involvement. Arch Neurol 1980;37:379-80.

${ }^{8}$ Wong RG, Moss CW, Hochstein DPH, Arko RJ, Schalla WO. Endotoxicity of the Legionnaires' disease bacterium. Ann Int Med 1979;90:624-7.

${ }^{\circ}$ Cohen WJ, Cohen NH. Lithium carbonate, haloperidol, and irreversible brain damage. JAMA 1974; 230:1283-7.

${ }^{10}$ Baastrup RC, Hollnagel P, Sorenson R, Schou M. Adverse reactions in treatment with lithium carbonate and haloperidol. JAMA 1976;236:2645-6.

${ }^{11}$ Tupin JP, Schuller AB. Lithium and haloperidol incompatibility reviewed. Psych J Univ Ottawa 1978; 3:245-51.

12 Ottosson JO. Systemic biochemical effects of ECT. In: Fink M et al (eds). Psychobiology of Convulsive Therapy. Washington: VH Winston and Sons, 1974. 